\title{
Molecular Characterization of a thiJ-like Gene in Chinese Cabbage
}

\author{
Kyung-Jin Oh, Yong-Soon Park, Kyung-Ah Lee, Yong Je Chung and Tae-Ju Cho* \\ Division of Life Sciences, College of Natural Sciences, Chungbuk National University, Cheongju 360-763, Korea
}

Received 24 July 2003, Accepted 17 September 2003

\begin{abstract}
A cDNA clone for a salicylic acid-induced gene in Chinese cabbage (Brassica rapa subsp. pekinensis) was isolated and characterized. The cabbage gene encoding a protein of 392 amino acids contained a tandem array of two thiJ-like sequences. ThiJ is a thiamin biosynthesis enzyme that catalyzes the phosphorylation of hydroxymethylpyrimidine (HMP) to HMP monophosphate. Although the cabbage gene shows a similarity to bacterial thiJ genes, it also shares a similarity with the human DJ-1, a multifunctional protein that is involved in transcription regulation, male fertility, and parkinsonism. The cabbage thiJ-like gene is strongly induced by salicylic acid and a nonhost pathogen, Pseudomonas syringae pv. tomato, which elicits a hypersensitive response in Chinese cabbage. Treatment of the cabbage leaves with BTH, methyl jasmonate, or ethephon showed that the cabbage thiJ-like gene expression is also strongly induced by BTH, but not by methyl jasmonate or ethylene. This indicates that the cabbage gene is activated via a salicylic acid-dependent signaling pathway. Examination of the tissue-specific expression revealed that the induction of the cabbage gene expression by BTH occurs in the leaf, stem, and floral tissues but not in the root.
\end{abstract}

Keywords: Brassica rapa, Chinese cabbage, Defense-related, thiJ-like

\section{Introduction}

Like animals, plants also have defense mechanisms against various pathogens and pests, although the underlying mechanisms differ from vertebrate immune responses.

The nucleotide sequence data that is reported in this paper was submitted to the GenBank nucleotide sequence database, and assigned the accession number AY335489.

*To whom correspondence should be addressed.

Tel: 82-43-261-2309; Fax: 82-43-267-2306

E-mail: tjcho@cbucc.chungbuk.ac.kr
Salicylic acid is a messenger molecule in the activation pathway of one of the disease-resistance responses, known as systemic acquired resistance (SAR). SAR is locally induced by pathogen or pest attack and spreads systemically, resulting in protection of the whole plant. SAR is also broad-spectrum and long-lasting in effect (Ryals et al., 1996). Defense responses are triggered upon the perception of invading pathogens by specific receptors that are encoded by disease resistance genes (Bent et al., 1996; Dangl and Jones, 2001). This recognition in turn activates a complex array of signaling pathways in plant cells. Many of the signaling components, such as EDS1, NDR1 and NPR1, have been studied by mutant analysis (Delaney, 1997; Feys and Parker, 2000). Biochemical studies have shown that reactive oxygen species and nitric oxide are important signaling molecules in the activation of disease resistance. These studies have also shown that MAP kinase cascades play a critical role in plant defense responses, as in animals (McDowell and Dangl, 2000; Nuerenberger and Scheel, 2001).

Now, a picture of defense mechanisms in plants is emerging. However, much research is still necessary in order to clarify uncertainties and ambiguities. Moreover, studies on defense mechanisms have focused on a limited number of model plants, such as Arabidopsis thaliana, tomato, and tobacco. Although the information obtained from these model plants has advanced our understanding of defense responses in plants as a whole, certain plants may have unique resistance mechanisms. The genus Brassica includes many important vegetable crops, such as broccoli, cabbage, Chinese cabbage, cauliflower, mustard, rape, kale, and turnip. Although these Brassica species have served as good model plants to study self-incompatibility (Takasaki et al., 2000), studies on defense mechanisms in Brassica have not been very active. Progress on identifying defense mechanisms in Chinese cabbage (Brassica rapa subsp. pekinensis), an important vegetable crop in Asia, has also been very slow, and few defense-related genes in Chinese cabbage have been characterized.

Previously, we isolated a partial cDNA clone that contained a Chinese cabbage thiJ-like gene exhibiting strong induction by salicylic acid and a pathogen (Park et al., 2003). Here, we report the cDNA sequence of the full coding region and more 
detailed expression profiles of the Chinese cabbage thiJ-like gene.

\section{Materials and Methods}

Plant materials and chemical treatments Brassica rapa subsp. pekinensis (cultivar Norang) seedlings were grown on potting compost after germination. Unless stated otherwise, the experiments were performed with cabbage seedlings at the seven- or eight-leaf stage. For the salicylic acid treatment, fully developed and healthy leaves from the plants were cut into $1 \times 1 \mathrm{~cm}$ pieces, and floated onto a $20 \mathrm{mM}$ MOPS buffer ( $\mathrm{pH} 7.5$ ) containing either $5 \mathrm{mM}$ or no salicylic acid (Sigma Chemical Co., St. Louis, USA) in a $10 \mathrm{~cm}$ or $15 \mathrm{~cm}$ petri dish. The leaf samples were then transferred to a growth chamber and incubated at $25^{\circ} \mathrm{C}$ under continuous fluorescent light. After 1-3 days of treatment, the leaf squares were harvested, weighed, and frozen immediately in liquid nitrogen. Next, $0.3 \mathrm{mM}$ benzothiadiazole (BTH), $1 \mathrm{mM}$ methyl jasmonate (Me-JA) in $0.1 \%$ [v/v] ethanol, $1 \mathrm{mM}$ ethephon, or $0.1 \%$ ethanol were sprayed on leaves of the intact plants. BTH $(5 \%$ active ingredient in wettable powder) was a kind gift from Novartis, Korea. Me-JA and ethephon were purchased from Aldrich Chemical Co. (Milwaukee, USA) or Sigma Chemical Co. (St. Louis, USA). For an analysis of the tissuespecific expression, the seeds were vernalized for three weeks at $4^{\circ} \mathrm{C}$ after sowing. Each germinated seed was then transferred to a vinyl pot $(9 \mathrm{~cm}$ in diameter) and grown until flowering. The aerial parts of the plants were treated with $0.3 \mathrm{mM}$ BTH by spraying. The roots were treated with BTH by pouring $200 \mathrm{ml}$ of $0.3 \mathrm{mM}$ BTH solution into each pot. The control plants were similarly treated with sterile distilled water.

Pathogen treatment Pseudomonas syringae pv. tomato (Pst) 259 was a kind gift from Dr. Cha at the Phytopathogenic Bacteriology Laboratory at Chungbuk National University, Korea. The Pseudomonas bacteria for inoculation were freshly grown on a NA agar medium ( $5 \mathrm{~g}$ of peptone, $3 \mathrm{~g}$ of beef extract, $2 \mathrm{~g}$ of yeast extract, and $15 \mathrm{~g}$ of Bacto-agar per liter) for one or two days at $30^{\circ} \mathrm{C}$. The bacteria were collected by gently scraping the colonies from the agar medium with a sterile glass rod after adding $2 \mathrm{ml}$ of sterile water per $85-\mathrm{mm}$ plate. After centrifugation at 5,000 $\times \mathrm{g}$, the bacterial pellet was resuspended in sterile water and adjusted to $\mathrm{OD}_{600}=0.1$. Cabbage leaves were inoculated with the bacterial suspension by syringe infiltration. Pst-treated cabbage plants were then transferred to a growth chamber and incubated at $25^{\circ} \mathrm{C}$ under continuous light. Visible hypersensitive response (HR) usually developed 24 to $36 \mathrm{~h}$ after inoculation. The control plants were similarly treated with sterile water.

cDNA library screening The thiJ-like gene was isolated from a Chinese cabbage cDNA library that was constructed with the Lambda ZapII vector using mRNA from cabbage leaves treated with salicylic acid. Aliquots of the library were grown on ten LB agar plates at the density of $2.5 \times 10^{4}$ pfu per plate and the phages were eluted with a SM buffer. The phage suspension from each plate was then examined by PCR for the presence of the thiJ-like clone. One of the PCR primers was a thiJ-like gene-specific primer: 5' CTTACCCTTGAGTAAACCATG 3'. The other primer was a vector sequence near the cloning site: 5' AACAGCTATGACCATG ATTACGCC 3'. The phages in the sub-library fraction yielding positive DNA bands were then diluted and grown on 24 plates at the density of 2,000 pfu per plate. Eluted phages from the plates were again examined by PCR.

To confirm whether the PCR-amplified DNA contained the thiJlike gene sequence, DNA blot hybridization was performed using the partial cDNA as a probe. The preparation of the alkaline phosphatase-conjugated DNA probe and chemiluminescent detection were carried out as described by Min et al. (2001) using the AlkPhos Direct labeling and detection kit from Amersham Pharmacia Biotech (Buckinghamshire, UK). Finally, the phage sample containing the largest insert was then screened by in situ plaque hybridization. Subcloning of the cDNA insert was done by in vivo excision of the pBluescript SK phagemid DNA with ExAssist helper phage, as described in Stratagenes protocol. The obtained plasmid clone was designated pBrThi23.

DNA sequence analysis and database search The nucleotide sequence of the cDNA insert was determined with an Applied Biosystems automatic sequencer at the Macrogen Co. (Seoul, Korea) Conventional M13-forward and M13-reverse primers were initially used to determine the sequence. Based on the sequence information, two oligonucleotide primers for each strand of the cDNA insert were synthesized and used for further sequence analysis. The gene-specific sequencing primers were spaced at about 400-bp intervals. The database search and computation were performed at NCBI (National Center for Biotechnology Information) using the BLAST network service. Motif searches were made using the PROSITE database at http://expasy.hcuge.ch/ sprot/prosite.html. Signal peptide prediction was performed using TargetP (Emanuelsson et al., 2000) and http://www.cbs.dtu.dk/ services/TargetP/. A multiple sequence alignment was done with the CLUSTAL W software (Thompson et al., 1994) at http:// www.ebi.ac.uk.

RNA extraction and Northern analysis Total RNA was prepared from frozen plant materials using the "hot phenol" method of De Vries et al. (1988). For the Northern analysis, $10 \mu \mathrm{g}$ of total RNA was separated on a $1.0 \%$ formaldehyde-agarose gel and blotted onto a Hybond- $\mathrm{N}^{+}$nylon membrane (Amersham Pharmacia Biotech, Buckinghamshire, UK) using the standard capillary transfer method. After UV-crosslinking at $125 \mathrm{~mJ}$, the blots were hybridized using the DNA probe that was labeled with digoxigenin (DIG). Chemiluminescent detection of the hybridized probe was carried out as described by Kim et al. (2002). The probe DNA for detecting the cabbage thiJ-like mRNA was prepared by PCR amplification of the cDNA insert of pBrThi23. The amplified DNA was labeled with DIG by random priming reaction. The DNA probe for glyceraldehyde 3-phosphate dehydrogenase (GAPD) was obtained using a GAPD cDNA clone (GenBank accession no. AF536826). The DNA probes for the cabbage PR1a (GenBank accession no. AF528177) and CYP83B1 (GenBank accession no. AF528175) were prepared using the cDNA clones (CPL1 and CPE$\mathrm{T} 9$, respectively) that were isolated in our laboratory (Ryang et al., 2002). The DIG-labeling, hybridization, and chemiluminescent immunodetection were performed using kits from Roche Molecular Biochemicals (Mannheim, Germany) 
(A)

ct -2

ATGGCTTCATCTACTAAGACGGTTATGATCCCTATTGCTCACGGTACCGAGCCACTTGAAGCAGTGGCGATGATC 75

$\begin{array}{llllllllllllllllllllllllll}M & A & S & S & T & K & T & V & M & I & P & I & A & H & G & T & E & P & L & E & A & V & A & M & I & \end{array}$ ACCGTCTTGCGGAGAGGTGGTGCTGACGTGACGGTGGCGTCTGTCGAGGATAAAGTTGGCGTTGATGCTTGTCAT 150 $\begin{array}{llllllllllllllllllllllllll}T & V & L & R & R & G & G & A & D & V & T & V & A & S & V & E & D & K & V & G & V & D & A & C & H & 50\end{array}$ AACATCAAGATGGTCGCTGATACTCTCCTCTCCGATATCACCGACTCTATTTTCGACCTTATTGTTCTCCCCGGA 225

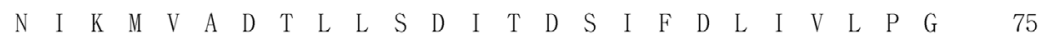
GGGCTTCCCGGAGGCGAGACTCTTAAAAACTGCAAACCATTAGAGAACATGGTGAAGAAACAAGACACAGATGGA 300 $\begin{array}{llllllllllllllllllllllllll}G & L & P & G & G & E & T & L & K & N & C & K & P & L & E & N & M & V & K & K & Q & D & T & D & G & 100\end{array}$ AGACTTAACGCAGCTATCTGTTGTGCTCCTGCTTTGGCTCTTGGTACTTGGGGTCTTCTCGAGGGCAAAACAGCA 375

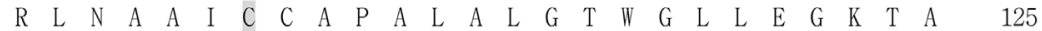
ACGGGTTACCCGGTTTTCATGGAGAAACTTGCAGCTACTTGTGCAACTGCAAGTGAATCAAGAGTTGAGATAGAT 450 $\begin{array}{llllllllllllllllllllllllll}T & G & Y & P & V & F & M & E & K & L & A & A & T & C & A & T & A & S & E & S & R & V & E & I & D & 150\end{array}$ GGAAGGATTGTGACAAGTCGAGGACCAGGAACCACCATTGAATTCTCCATCACTCTTATTGAGCAGTTGTTTGGT 525 $\begin{array}{llllllllllllllllllllllllll}G & R & I & V & T & S & R & G & P & G & T & T & I & E & F & S & I & T & L & I & E & Q & L & F & G & 175\end{array}$ AAAGACAAAGCTGATGAAGTCTCTAGTGGCTTGCTGGTTCGTCCTAATCCTGGTGAGGAGTTTACCTTTACCGAG 600 $\begin{array}{llllllllllllllllllllllllll}K & D & K & A & D & E & V & S & S & G & L & L & V & R & P & N & P & G & E & E & F & T & F & T & E & 200\end{array}$ CTTAACCAAACGAACTGGTCATTCCAAGATACTCCACAGATTCTCGTTCCCATTGCAGAGGACTCAGAGGAAATT 675 $\begin{array}{llllllllllllllllllllllllll}\text { L } & N & \text { Q } & \text { T } & \text { N } & \text { W } & S & F & \text { Q } & \text { D } & \text { T } & \text { P } & \text { Q } & \text { I } & \text { L } & \text { V } & \text { P } & \text { I } & \text { A } & \text { E } & \text { D } & \text { S } & \text { E } & \text { E } & \text { I } & 225\end{array}$ GAAGCTATAGCGCTTGTAGATATCCTCAGGAGAGCAAAAGCAAACGTCGTGATAGCTGCAGTTGGTAACAGTTTG 750

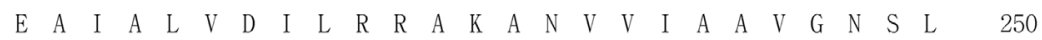
GAAGTTGTAGGATCTCGCAAAGCTAAGTTAGTAGCAGATGTGCTTCTTGATGAAGTTGCAGAGAAGTCGTTTGAT 825 $\begin{array}{llllllllllllllllllllllllll}\text { E } & \text { V } & \text { V } & \text { G } & \text { S } & \text { R } & \text { K } & \text { A } & \text { K } & \text { L } & \text { V } & \text { A } & \text { D } & \text { V } & \text { L } & \text { L } & \text { D } & \text { E } & \text { V } & \text { A } & \text { E } & \text { K } & \text { S } & \text { F } & \text { D } & 275\end{array}$ CTGATTGTGTTGCCTGGAGGTCTTAACGGTGCACCAAGATTGGCAAGTTGCGAGAAATTGGTGATATGTTAAAG 900 $\begin{array}{llllllllllllllllllllllllll}\text { L } & \text { I } & \text { V } & \text { L } & \text { P } & G & G & \text { L } & \text { N } & G & \text { A } & \text { P } & R & \text { L } & \text { A } & \text { S } & C & \text { E } & K & \text { L } & \text { V } & \text { N } & \text { M } & \text { L } & \text { K } & 300\end{array}$ AAACAAGCTGAAGCAAACAAACCTTATGGAGGAATATGCGCATCGCCTGTTTACGTCTTTGAGCCTCATGGTTTA 975

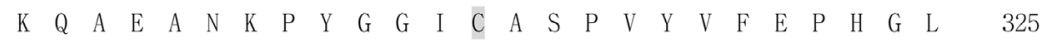
CTCAAGGGTAAGAAGGCAACTACACACCCATGTGTGAGCAACAAACTTTCGGACCAGAGCCACGTTGAGCACAGA 1050 $\begin{array}{llllllllllllllllllllllllll}\text { L } & K & G & K & K & A & T & T & H & P & C & V & S & N & K & L & S & D & Q & S & H & V & E & H & R & 350\end{array}$ GTTGTTGTGGATGGAAATGTGATAACGAGCAGAGCTCCAGGGACTGCAATGGAGTTTTCACTTGCGATTGTTGAG 1125 $\begin{array}{llllllllllllllllllllllllll}\text { V } & \text { V } & \text { V } & \text { D } & G & \text { N } & \text { V } & \text { I } & \text { T } & \text { S } & R & \text { A } & \text { P } & G & \text { T } & \text { A } & \text { M } & \text { E } & \text { F } & \text { S } & \text { L } & \text { A } & \text { I } & \text { V } & \text { E } & 375\end{array}$ AAGTTTTACGGGCGAGAGAAAGCGGTTCAGCTCGCCAAGGCAACACTTGTGTAAat tcaaaagt atgaaatgtgg 1200

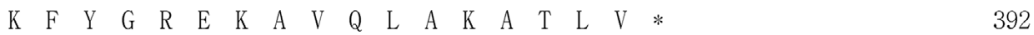
agaggctgcaagggacgtgat aaaaggggt tt tatgtatctttctgt tgatgatgatgagtgact t tgt aatgtg 1275 agcat tggctgaatggagtgtgaattttatgtctttaat aaatgaat aaacaaaggt taaaaaaaaaaaaaa 1350 аaаaаaаaа

(B)

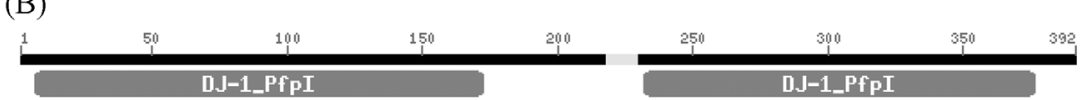

Fig. 1. A thiJ-like gene in Chinese cabbage. (A) Nucleotide and deduced amino acid sequences. The 5' and 3 ' noncoding cDNA sequences are represented by lowercase letters. The putative catalytic cysteine residues (C107 and C313) are shaded. (B) Schematic representation of the gene structure. DJ-1 is a multifunctional human protein involved in transcription activation, male fertility, and parkinsonism. PfpI represents an intracellular cysteine protease from Pyrococcus furiosus. The Pfam accession number of the DJ-1/PfpI family is PF01965. The gray region (\#218-229 in the sequence) represents a low-complexity segment that was filtered out in the BLASTP conserved domain search.

\section{Results}

\section{Isolation and characterization of the Chinese cabbage} thiJ-like gene To obtain a cDNA clone for the salicylic acid-induced thiJ-like gene in Chinese cabbage, a cDNA library was screened by PCR and in situ plaque hybridization, as described in Materials and Methods. The cDNA clone, designated pBrThi23, contained a 1,176-bp ORF with 5 ' and 3 ' noncoding sequences of 2-bp and 155-bp, respectively (Fig. 1A). The Chinese cabbage gene encoded a protein of 392 amino acids. Scanning the PROSITE database revealed no significant motif in the cabbage protein. A BLAST search showed that the cabbage protein was $92 \%$ identical to an Arabidopsis ThiJ-like protein of unknown function, which was encoded by AT3g14990. ThiJ was a thiamin biosynthesis enzyme that catalyzed the phosphorylation of hydroxymethylpyrimidine (HMP) to HMP monophosphate. The enzyme was primarily studied in E. coli (Mizote et al., 1999), but has not been characterized in plants. There are two other Arabidopsis proteins that are highly homologous to the cabbage protein (BLASTP value $\mathrm{E}<10^{-70}$ ): these two Arabidopsis proteins that are encoded by At1g53280 and 


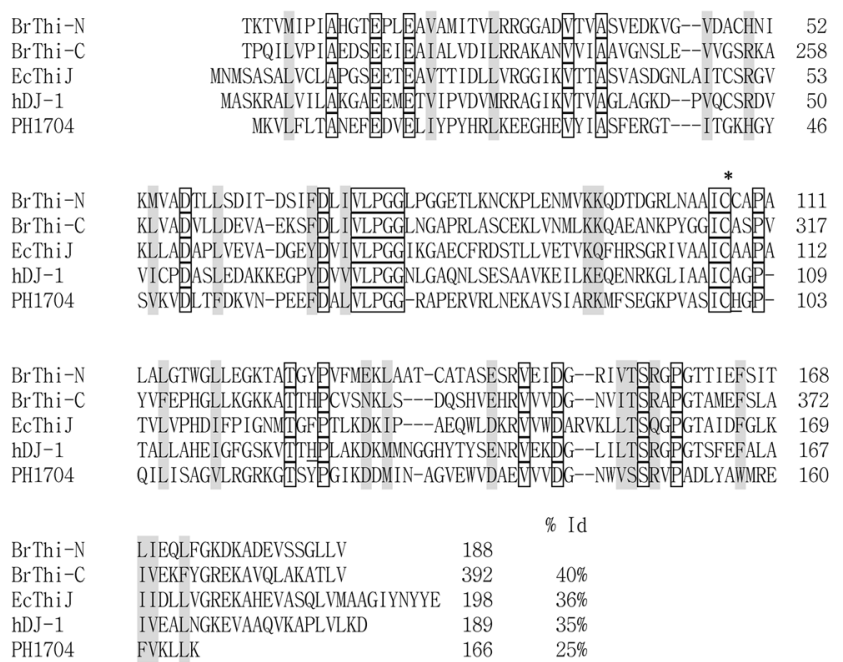

Fig. 2. Multiple sequence alignment of the two ThiJ-like domains of the cabbage protein and other homologous proteins. Identical amino acids are boxed, and conserved amino acids or amino acids with similar properties are shaded. An asterisk indicates the catalytic cysteine residue. The catalytic histidine residues in DJ-1 (H126) and PH1704 (H101) are underlined. BrThi-N, N-terminal ThiJ-like domain of the cabbage protein (amino acids \#5-188); Br-Thi-C, C-terminal domain of the cabbage protein (amino acids \#211-392); hDJ-1, human DJ-1 (Accession no. NP_009193); EcThiJ, ThiJ from E. coli (Accession no. Q46948); PH1704, protease I from Pyrococcus horikoshii (Accession no. NP_143548).

At4g34020 display a 79\% and 43\% identity, respectively, with the cabbage protein. The function of these Arabidopsis proteins has not yet been determined.

A search for conserved domains revealed the presence of two putative domains of a DJ-1/PfpI family, formerly known as a ThiJ/PfpI family. This structural feature is schematically represented in Fig. 1B. The two predicted ThiJ-like domains of the cabbage protein and other homologous proteins are compared by a CLUSTAL $\mathrm{W}$ analysis in Fig. 2. The Nterminal ThiJ-like domain (amino acids \#5-188) showed a $40 \%$ identity and a $63 \%$ similarity with the C-terminal domain (\#211-392). Compared to the E. coli ThiJ, the Nterminal ThiJ-like domain of the cabbage protein displayed a $36 \%$ identity and a 57\% similarity. The C-terminal domain showed a $35 \%$ identity and a $54 \%$ similarity with the bacterial enzyme.

As shown in Fig. 2, the cabbage protein also displayed a comparable similarity to the human protein DJ-1, which was reported to be involved in male fertility (Wagenfeld et al., 1998; Takahashi et al., 2001) and Parkinson's disease (Bonifati et al., 2003). The crystal structure of the DJ-1 was recently elucidated (Honbou et al., 2003). The structure analysis showed that DJ-1 was structurally most similar to the monomer unit of protease I (PH1704) from the hyperthermophilic archaebacterium Pyrococcus horikoshii (Du et al., 2000). PH1704 is a homolog of the novel

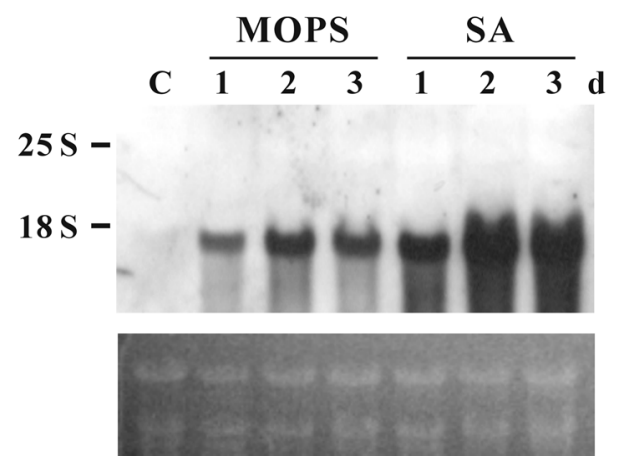

Fig. 3. Induction of the cabbage thiJ-like gene expression by salicylic acid. Chinese cabbage leaves were cut into $1 \times 1 \mathrm{~cm}$ squares and floated onto a $20 \mathrm{mM}$ MOPS buffer (pH 7.5) containing $5 \mathrm{mM}$ salicylic acid. Control samples were treated with the MOPS buffer without salicylic acid. C, control leaf sample with no treatment; MOPS, $1 \times 1 \mathrm{~cm}$ leaf squares treated with MOPS buffer; SA, leaf squares treated with salicylic acid. 1,2 , and 3 represent the duration of the treatment in days. After the indicated time, the leaf samples were collected and analyzed by Northern blot hybridization. Next, $10 \mu \mathrm{g}$ of the total RNA from each sample was size-fractionated on a $1 \%$ formaldehyde agarose gel. The agarose gel, stained with ethidium bromide, is represented in the lower panel. The fractionated RNA was blotted onto a nylon membrane and hybridized with a DIGlabeled DNA probe. Chemiluminescent detection was done using an alkaline phosphatase-conjugated anti-DIG antibody and CSPD, according to the protocol provided by Roche Molecular Biochemicals.

intracellular cysteine protease PfpI that is found in P. furiosus (Halio et al., 1996). As shown in Fig. 2, cabbage protein also shows a considerable similarity to the $\mathrm{PH} 1704$ protease. The putative catalytic cysteine residue that is conserved in DJ-1 and PH1704 is also present in the two ThiJ-like domains of the cabbage protein (C107 and C313, respectively). In papain, an archetype of the cysteine protease family, the thiolate ion acts as an attacking nucleophile and is stabilized through the formation of an ion pair with neighboring imidazolium group of a histidine residue (Dardenne et al., 2003). The putative histidine residue in the active site (H334) is also conserved in the C-terminal ThiJ-like domain, as in DJ-1. The catalytic histidine residue, however, is not evident in the N-terminal domain.

Expression patterns of the cabbage thiJ-like gene To examine whether the expression of the Chinese cabbage thiJlike gene is induced by salicylic acid, a Chinese cabbage leaf was cut into $1 \times 1 \mathrm{~cm}$ pieces and treated with a MOPS buffer containing $5 \mathrm{mM}$ salicylic acid. As shown in Fig. 3, the salicylic acid treatment strongly induced the expression of the cabbage thiJ-like gene. The mock treatment without salicylic acid also induced the gene expression, indicating that the gene is inducible by wounding.

Our research then examined whether the gene would be induced by the stimuli that accompany hypersensitive 


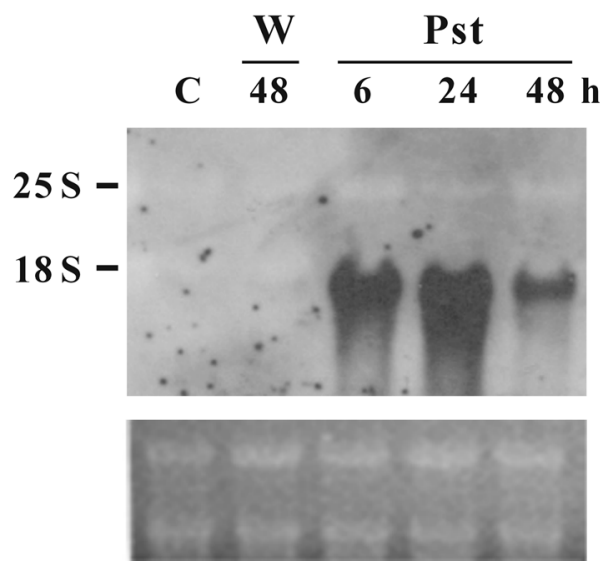

Fig. 4. Induction of the cabbage thiJ-like gene expression by pathogen. Chinese cabbage leaves were infiltrated with either sterile water (W) or Pseudomonas syringae pv. tomato (Pst). C and W48 represent the control leaf sample with no treatment and the leaf sample harvested $48 \mathrm{~h}$ after infiltration with sterile water, respectively. After the indicated time, the leaf samples were collected and analyzed by Northern blot hybridization (Fig. 3.)

response (HR), since HR is often a prerequisite for induction of disease resistance in plants. We used Pseudomonas syringae pv. tomato (Pst) to induce HR in Chinese cabbage. Pst causes bacterial speck disease in tomatoes and Arabidopsis (Bashan et al., 1981; Whalen et al., 1991), but not in Chinese cabbage (Ryang et al., 2002). In the Pstinfiltrated cabbage leaves, visible necrosis was evident 24-36 $\mathrm{h}$ after inoculation. As shown in Fig. 4, the Northern analysis showed that the gene was strongly induced in the cabbage leaves that were infiltrated with Pst. In contrast, the induction of the gene expression was not observed in the control leaves that were infiltrated with water.

Chemicals other than salicylic acid can also activate defense reactions. One of the most potent SAR-inducing chemicals is benzothiadiazole (Goerlach et al., 1996). To see if the cabbage thiJ-like gene is also induced by benzothiadiazole $(\mathrm{BTH})$, the cabbage leaves were sprayed with $0.3 \mathrm{mM}$ BTH and analyzed by Northern blot hybridization. The result showed that the cabbage gene was also strongly induced by BTH (Fig. 5). This confirms the result that was obtained with salicylic acid, since BTH is known to be a salicylic acid-mimicking chemical. We then examined the effect of other defense response activators, jasmonate and ethylene, since plant defense responses can also be activated via salicylic acid-independent signaling pathways that are mediated by jasmonates and ethylene (Piterse and van Loon, 1999). To see how the cabbage gene responds to ethylene or jasmonates, the cabbage leaves were treated with methyl jasmonate (Me-JA) or with ethephon, an ethylene-releasing compound. As shown in Fig. 5, the cabbage thiJ-like gene was not induced either by ethylene or by Me-JA.

To verify that appropriate induction conditions were used,

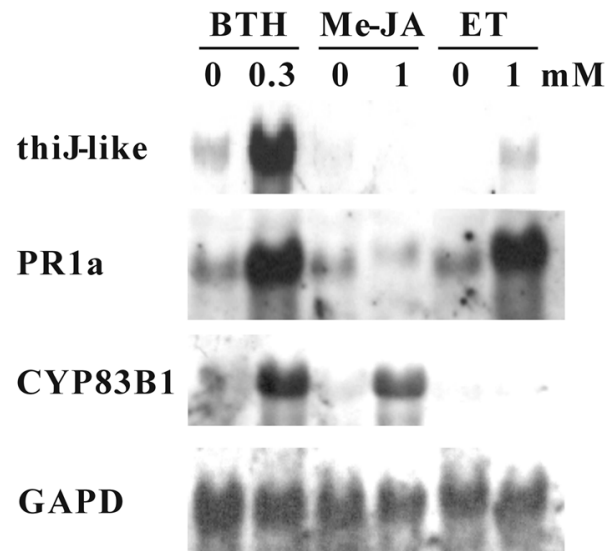

Fig. 5. Induction by BTH, methyl jasmonate, and ethylene. 0.3 $\mathrm{mM}$ of BTH, $1 \mathrm{mM}$ methyl jasmonate (Me-JA) in $0.1 \%$ ethanol, $1 \mathrm{mM}$ ethephon solution, or $0.1 \%$ ethanol and sterile water (control) was sprayed onto cabbage leaves. After $24 \mathrm{~h}$, the leaf samples were collected and analyzed by Northern blot hybridization using DIG-labeled probes (Fig. 3). The transcript levels of the cabbage PR1a (CPL1) and CYP83B1 (CPE-T9) genes were assayed as positive controls for the induction by ethylene and Me-JA, respectively. As a loading control, the samples were also hybridized with the cDNA specific for the cabbage glyceraldehyde-3-phosphate dehydrogenase (GAPD).

we included as positive controls the expression of the Me-JAinducible Chinese cabbage gene CPE-T9 and the ethyleneinducible gene CPL1, which were isolated in our laboratory as Pst-induced genes (Ryang et al., 2002). The CPE-T9 gene encodes a putative CYP83B1, an oxime-metabolizing enzyme in the glucosinolate biosynthesis (Hansen et al., 2001). The cabbage cytochrome $\mathrm{P} 450$ gene was induced by both BTH and Me-JA (Fig. 5). The CPL24-1 that was used as a positive control for induction by ethylene encodes a PR1a. Figure 5 shows that the cabbage PR1a gene is induced by both BTH and ethylene. These results, therefore, clearly show that the cabbage thiJ-like gene is induced by salicylic acid or BTH, but not by Me-JA or ethylene.

We then examined the tissue specificity of the gene expression. To this end, cabbage plants were induced to bolt. After flowering, the whole plants were treated with BTH by spraying (aerial parts) and soaking (roots) with a $0.3 \mathrm{mM}$ BTH solution. As shown in Fig. 6, the gene was weakly expressed in all of the tissues that were examined without the BTH treatment. The result also showed that the strong induction by BTH occurred in the leaf, stem, and mature flower, but not in the root and flower bud.

\section{Discussion}

There have only been a few reports on thiamin biosynthesis in plants, except the report by Kim et al. (1998) that described a Brassica napus gene, which encodes a bifunctional enzyme 


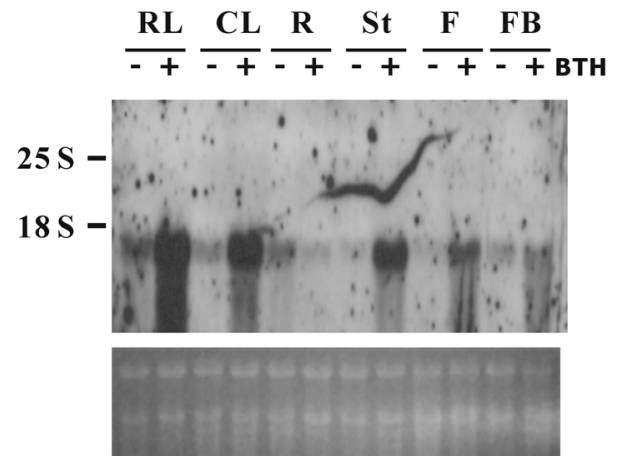

Fig. 6. Tissue-specific expression of the cabbage thiJ-like gene. Chinese cabbage plants were induced to bolt by vernalizing the seeds. The cabbage plants bearing flowers were treated with BTH by spraying (aerial parts) and soaking (roots) the plants with a $0.3 \mathrm{mM}$ BTH solution. The control plants were similarly treated with sterile water. Rosette leaf (RL), cauline leaf (CL), root $(\mathrm{R})$, stem $(\mathrm{ST})$, mature flower $(\mathrm{F})$, and flower bud (FB) tissues were collected from the plants and analyzed by Northern blot hybridization using DIG-labeled DNA probe (Fig. 3).

that has 2-methyl-4-amino-5-hydroxypyrimidine-monophosphate kinase (HMP-P kinase) and thiamine-phosphate pyrophosphorylase (TMP-PPase) activities. There has been no report on the HMP kinase (ThiJ) enzyme in plants. A BLAST search with the $E$. coli ThiJ as a query showed that the most homologous Arabidopsis protein to the E. coli ThiJ was a putative protein that was encoded by AT1g53280, which is $40 \%$ identical to the bacterial enzyme. This Arabidopsis protein is the same protein that shows a $79 \%$ identity with the cabbage ThiJ-like protein. The second most homologous Arabidopsis protein to the E. coli ThiJ was encoded by AT3g14990, which also displayed a 92\% identity with the cabbage thiJ-like gene at the amino acid level. Therefore, there seems to be a good possibility that the cabbage gene that was isolated in this study represents the thiJ gene that encodes a HMP kinase.

Thiamin biosynthesis may occur in plastid. Consistent with this, the bifunctional protein that has HMP-P kinase and TMP-PPase activities has a transit peptide that directs it to chloroplast. To examine whether the cabbage ThiJ-like protein has the N-terminal transit peptide, a signal peptide prediction was performed using TargetP (Emanuelsson et al., 2000). However, a transit peptide was not evident in the cabbage protein. The protein was predicted to have neither the signal peptide for secretory pathway nor the signal for mitochondrial transport. If this prediction is correct, then the result suggests that the cabbage protein is cytoplasmic, which is inconsistent with the role of the protein in thiamin biosynthesis. Thus, although the cabbage protein is homologous to the bacterial ThiJ, it is still unclear whether it is indeed a HMP kinase.

The cabbage protein shows similarities to the DJ-1 and the cysteine protease, $\mathrm{PH} 1704$. The fact may indicate a function that is different from the HMP kinase. Although the exact function is currently unknown, the information on structure and function of the multifunctional DJ-1 is intriguing. DJ-1 was originally reported to show cooperative transforming activity with H-ras (Nagakubo et al., 1997). Later, DJ-1 was shown to be a multifunctional protein that plays essential roles in the testis and brain. DJ-1 was reported to be related to male fertility, and its level was reduced in rat sperm that was treated with sperm toxicants that cause infertility in rats (Wagenfeld et al., 1998). It was also found that DJ-1 positively regulates the androgen receptor by impairing the binding of PIASx $\alpha$ to the receptor (Takahashi et al., 2001). Additionally, a recent report showed that a mutation of DJ-1 is responsible for familial Parkinson's disease (Bonifati et al., 2003). A recent structural analysis by Honbou et al. (2003) revealed that DJ-1 contains an additional $\alpha$-helix at the $\mathrm{C}$-terminal region, which blocks the putative catalytic site of DJ-1 and appears to regulate enzymatic activity. Considering that the DJ-1 expression is induced by oxidative stress, such as $\mathrm{H}_{2} \mathrm{O}_{2}$ or paraquat, the authors suggest that DJ-1 may induce conformational changes to acquire protease activity in response to oxidative stress.

It is interesting that DJ-1 is induced by oxidative stress (such as $\mathrm{H}_{2} \mathrm{O}_{2}$ ), since $\mathrm{H}_{2} \mathrm{O}_{2}$ is rapidly generated by NADPH oxidase at the site of pathogen infection in plants (HammondKosack and Jones, 1996). Subsequently, salicylic acid and nitric oxide accumulate and synergistically act to induce localized cell death (hypersensitive response) and other defense responses (McDowell and Dangl, 2000). Thus, although it is still unclear whether the cabbage protein is functionally and structurally related to DJ-1, it is tempting to speculate that the protease activity of the cabbage ThiJ-like protein is activated by pathogen infection, possibly via salicylic acid, and regulates downstream defense responses. Recently, reports on the role of cysteine proteases in plant defense have accumulated. A cysteine protease was reported to be required for Cf-2-dependent disease resistance (Krueger et al., 2002). Cysteine proteases and their proteinaceous inhibitors were also reported to regulate hypersensitive cell death (Belenghi et al., 2003).

DJ-1 protein exists as a dimer (Honbou et al., 2003). This dimer formation appears to be essential for the DJ-1 protein to properly function, since a DJ-1 mutation that is associated with human parkinsonism (Bonifati et al., 2003) disrupts the hydrophobic interactions between monomers. Likewise, the PH1704 protease activity is exhibited only in the oligomeric forms of the protein ( $\mathrm{Du}$ et al., 2000). The dimeric or oligomeric nature of these proteins may answer the questions of why the cabbage gene has sequence duplication. It remains to be elucidated whether the two ThiJ-like domains are functionally related to DH-1 or ThiJ. Whatever the function is, it will be interesting to see how the N-terminal and C-terminal domains of the cabbage protein interact with each other to form an active protein.

Acknowledgments This work was supported by a grant 
from the Technology Development Program of the Ministry of Agriculture and Forestry, Republic of Korea (Grant no. 299045-3).

\section{References}

Bashan, Y., Sharon, E., Okon, Y. and Henis, Y. (1981) Scanning electron and light microscopy of infection and symptom development in tomato leaves infected with Pseudomonas tomato. Physiol. Plant Pathol. 19, 139-144.

Belenghi, B., Acconcia, F., Trovato, M., Perazzolli, M., Bocedi, A., Polticelli, F., Ascenzi, P. and Delledonne, M. (2003) AtCYS1, a cystatin from Arabidopsis thaliana, suppresses hypersensitive cell death. Eur. J. Biochem. 270, 2593-2604.

Bent, A. F. (1996) Plant disease resistance genes: Function meets structure. Plant Cell 8, 1757-1771.

Bonifati, V., Rizzu, P., Van Baren, M. J., Schaap, O., Breedveld, G. J., Krieger, E., Dekker, M. C., Squitieri, F., Ibanez, P., Joose, M., van Dongen, J. W., Vanacore, N., Van Swieten, J. C., Brice, A., Meco, G., van Duijn, C. M., Oostra, B. A. and Heutink, P. (2003) Mutations in the DJ-1 gene associated with autosomal recessive early-onset parkinsonism. Science 299, 256-259.

Dangl, J. L. and Jones, J. D. G. (2001) Plant pathogens and integrated defence responses to infection. Nature 411, 826-833.

Dardenne, L. E., Werneck, A. S., De Oliviera Neto, M. and Bisch, P. M. (2003) Electrostatic properties in the catalytic site of papain: A possible regulatory mechanism for the reactivity of the ion pair. Proteins 52, 236-253.

De Vries, S., Hoge, H. and Bisseling, T. (1988) Isolation of total and polysomal RNA from plant tissues; in Plant Molecular Biology, S.B. Gelvin and R.A. Schilperoot (eds), pp. B6/1-5, Kluwer Academic Publishers, Dordrecht, The Netherlands.

Delaney, T. P. (1997) Genetic dissection of acquired resistance to disease. Plant Physiol. 113, 5-12.

Du, X., Choi, I.-G., Kim, R., Wang, W., Jancarik, J., Yokota, H. and Kim, S.-H. (2000) Crystal structure of an intracellular protease from Pyrococcus horikoshii at 2-A resolution. Proc. Natl. Acad. Sci. USA 97, 14079-14084.

Emanuelsson, O., Nielsen, H., Brunak, S. and von Heijne, G. (2000) Predicting subcellular localization of proteins based on their N-terminal amino acid sequence. J. Mol. Biol. 300, 10051016.

Feys, B. and Parker, J. E. (2000) Interplay of signaling pathways in plant disease resistance. Trends Genet. 16, 449-455.

Goerlach, J., Volrath, S., Knauf-Beiter, G., Hengy, G., Beckhove, U., Kogel, K.-H., Oostendorp, M., Staub, T., Ward, E., Kessmann, H. and Ryals, J. (1996) Benzothiadiazole, a novel class of inducers of systemic acquired resistance, activates gene expression and disease resistance in wheat. Plant Cell 8, 629643.

Halio, S. B., Blumentals, I. I., Short, S. A., Merrill, B. M. and Kelly, R. M. (1996) Sequence, expression in Escherichia coli, and analysis of the gene encoding a novel intracellular protease (PfpI) from the hyperthermophilic archaeon Pyrococcus furiosus. J. Bacteriol. 178, 2605-2612.

Hammond-Kosak, K. E. and Jones, J. D. G. (1996) Resistance gene-dependent plant defense responses. Plant Cell 8, 1773-
1791.

Hanfrey, C., Fife, M. and Buchanan-Wollaston, V. (1996) Leaf senescence in Brassica napus: Expression of genes encoding pathogenesis-related proteins. Plant Mol. Biol. 30, 597-609.

Hansen, C. H., Du, L., Naur, P., Olsen, C. E., Axelsen, K. B., Hick, A. J., Pickett, J. A. and Halkier, B. A. (2001) CYP83B1 is the oxime-metabolizing enzyme in the glucosinolate pathway in Arabidopsis. J. Biol. Chem. 276, 24790-24796.

Honbou, K., Suzuki, N. N., Horiuchi, M., Niki, T., Taira, T., Ariga, H. and Inagaki, F. (2003) The crystal structure of DJ-1, a protein related to male fertility and Parkinson's disease. $J$. Biol. Chem. 278, 31380-31384.

Kim, M., Lim, C.-J. and Kim, D. (2002) Transcription of Schizosaccharomyces pombe thioltransferase-1 in response to stress conditions. J. Biochem. Mol. Biol. 35, 409-413.

Kim, Y. S., Nosaka, K., Downs, D. M., Kwak, J. M., Park, D., Chung, I. K. and Nam, H. K. (1998) A Brassica cDNA clone encoding a bifunctional hydroxymethylpyrimidine kinase/ thiamin-phosphate pyrophosphorylase involved in thiamin biosynthesis. Plant Mol. Biol. 37, 955-966.

Krueger, J., Thomas, C. M., Golstein, C., Dixon, M. S., Smoker, M., Tang, S., Mulder, L. and Jones, J. D. G. (2002) A tomato cysteine protease required for Cf-2-dependent disease resistance and suppression of autonecrosis. Science 296, 744-747.

McDowell, J. M. and Dangl, J. L. (2000) Signal transduction in the plant immune response. Trends Biochem. Sci. 25, 79-82.

Min, H.-J., Park, S.-S. and Cho, T.-J. (2001) A simple and efficient subtractive cloning method. J. Biochem. Mol. Biol. 34, 59-65.

Mizote, T., Tsuda, M., Smith, D. D. S., Nakayama, H. and Nakazawa, T. (1999) Cloning and characterization of the thiD/J gene of Escherichia coli encoding a thamin-synthesizing bifunctional enzyme, hydroxymethylpyrimidine kinase/ phosphomethylpyrimidine kinase. Microbiology 145, 495-501.

Nagakubo, D., Taira, T., Kitaura, H., Ikeda, M., Tamai, K., IguchiAriga, S. M. M. and Ariga, H. (1997) DJ-1, a novel oncogene which transforms mouse NIH3T3 cells in cooperation with ras. Biochem. Biophys. Res. Commun. 231, 509-513.

Nuernberger, T. and Scheel, D. (2001) Signal transmission in the plant immune response. Trends Plant Sci. 6, 372-379.

Park, Y.-S., Min, H.-J., Ryang, S.-H., Oh, K.-J., Cha, J.-S., Kim, H.Y. and Cho, T.-J. (2003) Characterization of salicylic acidinduced genes in Chinese cabbage. Plant Cell Rep. 21, 10271034.

Pieterse, C. M. J. and van Loon, L. C. (1999) Salicylic acidindependent plant defence pathways. Trends Plant Sci. 4, 5258.

Ryals, J. A., Neuenschwander, U. H., Willits, M. G., Molina, A., Steiner, H. Y. and Hunt, M. D. (1996) Systemic acquired resistance. Plant Cell 8, 1809-1819.

Ryang, S.-H., Chung, S.-Y., Lee, S.-H., Cha, J.-S., Kim, H. Y. and Cho, T.-J. (2002) Isolation of pathogen-induced Chinese cabbage genes by subtractive hybridization employing selective adaptor ligation. Biochem. Biophys. Res. Commun. 299, 352359.

Takahashi, K., Taira, T., Niki, T., Seino, C., Iguchi-Ariga, S. M. and Ariga, H. (2001) DJ-1 positively regulates the androgen receptor by impairing the binding of PIASx alpha to the receptor. J. Biol. Chem. 276, 37556-37563.

Takasaki, T., Hatakeyama, K., Suzuki, G., Watanabe, M., Isogai, 
A. and Hinata, K. (2000) The S receptor kinase determines self-incompatibility in Brassica stigma. Nature 403, 913-916.

Thompson, J. D., Higgins, D. G. and Gibson, T. J. (1994) CLUSTAL W: improving the sensitivity of progressive multiple alignment through sequence weighting, position-specific gap penalties and weight matrix choice. Nucleic Acids Res. 22, 4673-4680.

Wagenfeld, A., Gromoll, J. and Cooper, T. G. (1998) Molecular cloning and expression of rat contraception associated protein 1 (CAP1), a protein putatively involved in fertilization. Biochem. Biophys. Res. Commun. 251, 545-549.

Whalen, M. C., Innes, R. W., Bent, A. F. and Staskawicz, B. J. (1991) Identification of Pseudomonas syringae pathogens of Arabidopsis and a bacterial locus determining avirulence on both Arabidopsis and soybean. Plant Cell 3, 49-59. 